\title{
René Audet, Nicolas Xanthos (dir.), Le roman contemporain au détriment du personnage
}

\section{Roberta Sapino}

\section{(2) OpenEdition}

1 Journals

\section{Edizione digitale}

URL: http://journals.openedition.org/studifrancesi/681

DOI: 10.4000/studifrancesi.681

ISSN: 2421-5856

\section{Editore}

Rosenberg \& Sellier

\section{Edizione cartacea}

Data di pubblicazione: 1 aprile 2015

Paginazione: 215-216

ISSN: 0039-2944

\section{Notizia bibliografica digitale}

Roberta Sapino, «René Audet, Nicolas Xanthos (dir.), Le roman contemporain au détriment du personnage », Studi Francesi [Online], 175 (LIX | I) | 2015, online dal 01 avril 2015, consultato il 18 septembre 2020. URL : http://journals.openedition.org/studifrancesi/681 ; DOI : https://doi.org/ 10.4000/studifrancesi.681

Questo documento è stato generato automaticamente il 18 settembre 2020.

\section{(c) $(1) \ominus$}

Studi Francesi è distribuita con Licenza Creative Commons Attribuzione - Non commerciale - Non opere derivate 4.0 Internazionale. 


\title{
René Audet, Nicolas Xanthos (dir.), Le roman contemporain au détriment du personnage
}

\author{
Roberta Sapino
}

\section{NOTIZIA}

RENÉ AUDET, NICOLAS XANTHOS (dir.), Le roman contemporain au détriment du personnage, Baltimore, Johns Hopkins University Press, 2014, «L’Esprit créateur», vol. 54, n. 1, pp. 151.

1 Nell'ampia e fiorente discussione sugli sviluppi del romanzo contemporaneo, osservano René AUDET e Nicolas XANTHOS nell'introduzione al volume («Le roman contemporain au détriment du personnage: introduction», pp. 1-7), la descrizione minuziosa delle nuove forme di narrazione non ha necessariamente coinciso con lo studio di un aspetto che pure ne è inscindibile: la costruzione di personaggi evanescenti, dai contorni labili e dalle declinazioni identitarie plurali, talvolta così privi di intenzionalità e psicologia da essere spodestati dal loro ruolo tradizionale di motore dell'azione. Proponendosi di indagare questo spazio ancora insufficientemente battuto, i due studiosi non intendono suggerire che la presenza di tali personaggi sia appannaggio esclusivo delle opere pubblicate nell'ultimo quarto di secolo: così come è sviante prendere alla lettera le affermazioni categoriche sulla sparizione del récit e sul suo recente ritorno in letteratura, allo stesso modo non bisogna ignorare che, anche nelle sue forme più strettamente autoreferenziali, il romanzo non smette di essere innanzitutto un discorso sull'esperienza umana. In quest'ottica, la scrittura contemporanea sarebbe caratterizzata non tanto dalla nascita ex nihilo di una volontà di problematizzazione e di gioco sul personaggio, quanto piuttosto dall'intensificazione e dalla diversificazione di una postura critica e autoriale già presente. 
2 Tre linee di ricerca distinte e tuttavia strettamente legate tra loro emergono dalla lettura degli undici contributi (alcuni dei quali redatti in inglese): la messa in discussione dell'unità dell'individuo, l'indebolimento dell'inserimento nel flusso della Storia, la possibilità (o l'impossibilità) di racchiudere la complessità umana entro i limiti del linguaggio.

Bruno BLANCKEMAN (Storytelling/storyfailing? Avatars du personnage dans le récit de fiction contemporain, pp. 8-21) inaugura la raccolta con un contributo di natura prettamente teorica nel quale ci si interroga sulla natura delle interazioni tra mentale e verbale, tra psichico e predicativo, e sul ruolo che esse esercitano nel processo di identificazione dei perimetri dell'umano. Dopo aver proposto la nozione di débordement come chiave di lettura del personaggio contemporaneo, lo studioso rintraccia due forme di elaborazione verbale della misura dell'umano (l'una satellitaire, l'altra sismographique), entrambe riconducibili a una rivalutazione critica dei concetti di humanisme e universalisme.

4 Marie-Hélène VOYER ( «Je suis devenu d'une méticulosité aberrante»: distorsions perceptives et discordances cognitives $d u$ personnage dans "Infiniment petit" de Patrick Chatelier et "Compression" de Nicolas Bouyssi, pp. 22-34) analizza due esempi emblematici di ciò che definisce il personnage discordant, figure di investigatori inefficaci e narratori inattendibili, caratterizzate da una certa volontà di auto-annullamento, che rimettono in causa la possibilità tanto di conoscere quanto di raccontare; una forma particolare di sparizione del personaggio romanzesco è poi messa in luce da Adeline CAUTE ( $« E t ~ m o i$, moi aujourd'hui»: disparition et dérive identitaires du personnage dans "V.O." d'Eva Almassy, pp. 35-46): nel primo libro di Eva Almassy, una soggettività ipertrofica invade la narrazione, facendo della negazione dell'altro la cifra dell'affermazione di sé e conducendo alla progressiva de-materializzazione del personaggio, in particolar modo di quello maschile.

5 Warren мотте (Nobody's Novel, pp. 47-57) si immerge tra i personaggi rarefatti di Villa Chagrin di Marie Cosnay, rivelandone il carattere arbitrario e contingente e portando l'attenzione sulla precarietà ontologica dello spazio della finzione in quanto opera del linguaggio; Alice RICHIR (À travers le masque: narrateur contraint et mise en récit dans “ L'Absolue Perfection du crime" et "Insoupçonnable", pp. 58-70) analizza due romanzi di Tanguy Viel e mostra come la ripresa dei meccanismi tipici del genere poliziesco consenta all'autore di elaborare una nuova forma di mise en récit attribuita a un testimone privo di parola propria ma reso puro ricettacolo dei racconti altrui; l'intervento di Nicolas XHANTOS (Renoncer à l'individu, dire l'humain: "Cent seize Chinois et quelques" comme fable de l'intime et du partagé, pp. 71-85) intende invece interrogarsi sulle conseguenze poetiche e antropologiche di un universo romanzesco che rinuncia ad ancorarsi alla nozione di individuo per aspirare a dire l'intimità costitutiva della comunità umana.

6 Il contributo di Eric LYNCH (Olivier Cadiot's Robinson, or A Portrait of the Artist as "Autousine", pp. 86-99) ripercorre i vari romanzi di Cadiot incentrati su Robinson, figura psicologicamente vuota che agisce come un vero e proprio motore atto a trattare freneticamente e macchinosamente sensazioni, percezioni, luoghi comuni, e per questo riconducibile al personnage conceptuel teorizzato da Deleuze e Guattari; l'oggetto elevato a fulcro della narrazione è invece al centro dell'intervento di Andrée MERCIER (Quand les objets parlent à la place du personnage: étude de "Wigrum" de Daniel Canty et de "Très-grande surface" d'André Benchetrit, pp. 100-114), attenta riflessione su due operazioni di 
scardinamento dell'idea di narrazione personale e della mediazione del soggetto come condizione d'esistenza dell'oggetto.

Dominique RAYMOND (Trois instances et un roman: "La Théorie de l'information" d'Aurélien Bellanger, pp. 115-125) si sofferma sul fenomeno mediatico della rentrée littéraire 2012, opera ibrida che alla biografia immaginaria di un manager informatico affianca una storia della tecnologia basata su frammenti di Wikipedia e che propone una rappresentazione della dinamica uomo-macchina basata sull'evoluzione discordante di un personaggio principale poco allettante, un narratore elevato a protagonista $\mathrm{e}$ un'eroina non antropomorfa; a seguire, Simon BROUSSEAU (Le nom propre en perte de sens: anonymisation du sujet chez Bruce Bégout, pp. 126-137) propone una lettura di Le ParK di Bruce Bégout in quanto caso eclatante di interrogazione sul ruolo del contesto sociale nei processi di regolazione degli individui e sulla graduale anonimizzazione di questi ultimi.

8 In chiusura, l'articolo di Linda AVENDAÑo ANGUITA ("Ici" de Nathalie Sarraute: une dynamique scopique, pp. 138-149) osserva come in Ici di Nathalie Sarraute il soggetto sia sottoposto a un processo di effacement - termine qui contrapposto a disparition -, per poi soffermarsi sulla complessità delle dinamiche scopiche e percettive che sottendono il romanzo.

9 Una nota bibliografica al testo di Philippe Bonnefis Une colère d'orgues: Pascal Quignard et la musique (Paris, Éditions Galilée, 2013) redatta da Bruno CHAOUAT è fornita in calce al volume. 